\title{
An orally active angiotensin-(1-7) inclusion compound and exercise training produce similar cardiovascular effects in spontaneously hypertensive rats
}

\author{
Mariane Bertagnolli ${ }^{\text {a,f }}$, Karina R. Casali ${ }^{a}$, Frederico B. De Sousa ${ }^{\text {b,f }}$, Katya Rigatto ${ }^{\mathrm{a}, \mathrm{c}}$, \\ Lenice Becker ${ }^{\mathrm{f}}$, Sergio H.S. Santos ${ }^{\mathrm{f}}$, Lucinara D. Dias ${ }^{\mathrm{a}}$, Graziela Pinto ${ }^{\mathrm{a}, \mathrm{c}}$, \\ Daniela R. Dartora ${ }^{a}$, Beatriz D. Schaan ${ }^{d}$, Ruben Dario Sinisterra Milan ${ }^{f}$, \\ Maria Claudia Irigoyen ${ }^{\mathrm{a}, e, \mathrm{f}}$, Robson A.S. Santos ${ }^{\mathrm{a}, \mathrm{f}, *}$ \\ a Instituto de Cardiologia do Rio Grande do Sul/Fundação Universitária de Cardiologia, 395 Av. Princesa Isabel, Porto Alegre, Rio Grande do Sul 90620-001, \\ Brazil \\ b Instituto de Física e Química, Universidade Federal de Itajubá, 1303 Av. BPS, Itajubá, Minas Gerais 37500-903, Brazil \\ ${ }^{c}$ Universidade Federal de Ciências da Saúde de Porto Alegre, 245 Rua Sarmento Leite, Porto Alegre, Rio Grande do Sul 90050-170, Brazil \\ ${ }^{\mathrm{d}}$ Endocrine Division, Hospital de Clínicas de Porto Alegre, and Department of Medicine, Faculty of Medicine, Universidade Federal do Rio Grande do Sul, \\ 2350 Rua Ramiro Barcelos, Porto Alegre, Rio Grande do Sul, 90035-003, Brazil \\ e Heart Institute/Universidade de São Paulo, 44 Av. Dr. Enéas de Carvalho Aguiar, São Paulo, São Paulo, 05403-900, Brazil \\ ${ }^{\mathrm{f}}$ INCT-Nanobiofar-Universidade Federal de Minas Gerais, 6627 Av. Antônio Carlos, Belo Horizonte, Minas Gerais 31270-901, Brazil
}

\section{A R T I C L E I N F O}

\section{Article history:}

Received 30 September 2013

Received in revised form

10 November 2013

Accepted 11 November 2013

Available online 18 November 2013

\section{Keywords:}

Hypertension

Renin-angiotensin system

Exercise training

Cardiac function

Autonomic control

\begin{abstract}
A B S T R A C T
Low angiotensin-(1-7) (Ang-(1-7)) concentration is observed in some cardiovascular diseases and exercise training seems to restore its concentration in the heart. Recently, a novel formulation of an orally active Ang-(1-7) included in hydroxy-propyl-beta-cyclodextrin (HPB-CD) was developed and chronically administered in experimental models of cardiovascular diseases. The present study examined whether chronic administration of HPB-CD/Ang-(1-7) produces beneficial cardiovascular effects in spontaneously hypertensive rats (SHR), as well as to compare the results obtained with those produced by exercise training. Male SHR (15-week old) were divided in control (tap water) or treated with HPB-CD/Ang-(1-7) (corresponding to $30 \mu \mathrm{g} \mathrm{kg}^{-1} \mathrm{day}^{-1}$ of Ang-(1-7)) by gavage, concomitantly or not to exercise training (treadmill, 10 weeks). After chronic treatment, hemodynamic, morphometric and molecular analysis in the heart were performed. Chronic HPB-CD/Ang-(1-7) decreased arterial blood pressure (BP) and heart rate in SHR. The inclusion compound significantly improved left ventricular (LV) end-diastolic pressure, restored the maximum and minimum derivatives $(\mathrm{d} P / \mathrm{d} T)$ and decreased cardiac hypertrophy index in SHR. Chronic treatment improved autonomic control by attenuating sympathetic modulation on heart and vessels and the SAP variability, as well as increasing parasympathetic modulation and HR variability. Overall results were similar to those obtained with exercise training. These results show that chronic treatment with the HPB-CD/Ang-(1-7) inclusion compound produced beneficial effects in SHR resembling the ones produced by exercise training. This observation reinforces the potential cardiovascular therapeutic effect of this novel peptide formulation.
\end{abstract}

(c) 2013 Elsevier Inc. All rights reserved.

\section{Introduction}

The renin-angiotensin system (RAS) is a major cardiovascular and renal control mechanism. Based on many recent studies this system is composed of two effector arms: the classical one angiotensin-converting enzyme (ACE)/angiotensin II (Ang

\footnotetext{
* Corresponding author at: Departamento de Fisiologia e Biofísica, ICB/UFMG, 6627, Antonio Carlos Avenue, Belo Horizonte, MG 31270-901, Brazil. Tel.: +55 31 34092956; fax: +55 3134092956 .

E-mail address: robsonsant@gmail.com (R.A.S. Santos).
}

II)/angiotensin II receptor type 1 (AT1) which is responsible for most of the known actions of the RAS and the counterregulatory arm ACE2/angiotensin-(1-7) (Ang-(1-7))/Mas receptor, which opposes a variety of the Ang II effects [10,11,39]. Ang-(1-7) is a heptapeptide primarily formed from angiotensin I by ACEindependent pathway and from angiotensin II cleavage by ACE2 [39]. Ang-(1-7) is present in the heart, and it contributes to preserve the cardiac function and the coronary perfusion in experimental models of cardiovascular diseases [22]. The cardioprotective effect of Ang-(1-7) is mainly mediated through G protein-coupled Mas receptor activation, also expressed in the heart, and modulated under physiological and pathological conditions [40]. Abnormal 
activation of the ACE/Ang II/AT1 receptor is observed in cardiovascular diseases, stimulating maladaptive cardiac responses and directly contributing to the progression to heart failure $[1,44]$.

The spontaneously hypertensive rat (SHR) has many similarities with the human essential hypertension, including high blood pressure (BP), cardiac remodeling and target-organ damage [43]. The ACE/Ang II/AT1 axis appears as a key mechanism stimulating cardiac hypertrophy and fibrosis in this model [37]. Cardiac structural changes are also accompanied by left ventricular (LV) dysfunction and the impairment of the autonomic control of the $\mathrm{BP}$ in this model $[4,20]$. The association of LV dysfunction, high BP and impaired autonomic control in SHR determines the progression to heart failure, and in humans, it is considered an important predictor of mortality and morbidity caused by cardiovascular diseases [21].

Beneficial effects of exercise training on managing high BP have been extensively described in humans [28,31] and animal models $[8,20]$ in the last decades. In SHR model, our group and others have previously shown that exercise training reduces BP mainly by improving the autonomic control and reducing sympathetic modulation in the heart and vessels [4], but also by decreasing overall cardiovascular oxidative stress and enhancing endothelial vasodilator function [3,33]. Although direct effects of exercise training on RAS are not well understood, some studies show upregulation of AT1 receptor in the heart after resistance training in normotensive rats [2], as well as increased Mas receptor expression and Ang-(1-7) concentration in SHR hearts after swim training [15]. In SHR, exercise training might therefore modulate the balance between both RAS axes which may contribute to prevent or reduce hypertension-induced cardiovascular dysfunction.

In addition to exercise, pharmacological approaches targeting the ACE/Ang II/AT1 axis are nowadays widely used in the treatment and handling of hypertension and heart failure. It is well established in the literature that AT1 receptor blockade in SHR reduces left ventricular (LV) mass and improves coronary hemodynamics [34]. However, the activation of the counteracting axis ACE2/Ang-(1-7)/Mas has been currently considered an alternative therapeutic strategy to control Ang II effects and to circumvent adverse responses observed with drugs blocking the Ang II axis. Thus, Mas agonists such as AVE 0991 or orally active formulations of Ang-(1-7) should be considered as a putative new class of cardiovascular drugs. Our group has recently described a new formulation of Ang-(1-7) which could allow the oral administration of this peptide, reducing its degradation by the digestive tract [23]. This is an inclusion compound composed of hydroxy-propyl-betacyclodextrin (HPB-CD), and Ang-(1-7). The structural arrangement of the inclusion compound protects the internal (guest) molecule, in this case Ang-(1-7), against proteolytic enzymes, and once HPB$\mathrm{CD}$ is enzymatically degraded by bacterial enzymes at the distal portions of the digestive tract it releases the peptide which is absorbed mainly in the colon. We therefore aim to assess in this study the potential therapeutic effect of the Ang-(1-7)-inclusion compound (HPB-CD/Ang-(1-7)) on BP values and heart function, as well as to compare its effects with the ones of exercise training on autonomic control of BP and heart rate (HR) in SHR. For this purpose, we hereby compare the effect of chronic administration of HPB-CD/Ang-(1-7) with exercise training, as well as the association of these two interventions.

\section{Methods}

\subsection{Animals}

Male SHR (15-week old, 250-300 g) were obtained from the Fundação Universitária de Cardiologia (Porto Alegre, Brazil) and divided in four experimental groups: sedentary treated with tap water $(n=8)$, sedentary treated with oral administration of HPB-CD/Ang-(1-7) inclusion compound ( $72 \mu \mathrm{g} \mathrm{kg}^{-1} \mathrm{day}^{-1}$, corresponding to $30 \mu \mathrm{g} \mathrm{kg}^{-1} \mathrm{day}^{-1}$ of Ang-(1-7))(n=8), exercise-trained treated with tap water $(n=9)$ and exercise-trained treated with HPB-CD/Ang-( $1-7)(n=8)$. Treatment was administered by gavage once per day during 10 weeks, in the morning, concomitantly with the exercise training protocol. Animals received standard laboratory chow and water ad libitum and were housed in temperature-controlled rooms $\left(22^{\circ} \mathrm{C}\right)$ with a 12 :12-h dark-light cycle. A moderate-intensity exercise protocol was performed in treadmill, 5 days/week during 10 weeks. Exercised rats progressed toward a speed of $20 \mathrm{~m} / \mathrm{min}$ for $1 \mathrm{~h}$, prescribed based on lactate threshold assessment, as previously described by Bertagnolli et al. [3]. One week before starting the exercise protocol, all animals were adapted to the procedure. All animal protocols and procedures used were in accordance with the Guidelines for Ethical Care of Experimental Animals and the US National Institutes of Health or European Commission guidelines, and were approved by Fundaçao Universitaria de Cardiologia de Porto Alegre Ethical Committee.

\subsection{Acute Ang-(1-7) absorption protocol}

Additional male SHR were allotted to an acute experiment to test the absorption of HPB-CD/Ang-(1-7) inclusion compound. Twentyfour hours before the experiment, a polyethylene catheter filled with saline was inserted in all rats femoral artery (under ketamine $90 \mathrm{mg} / \mathrm{kg}$ and xylazine $20 \mathrm{mg} / \mathrm{kg}$ anesthesia) to allow the collection of blood samples. SHR were divided in three groups ( $n=5 /$ group): control treated with tap water, treated with Ang-(1-7) not included (Bachem), and treated with HPB-CD/Ang-(1-7) inclusion compound $\left(72 \mu \mathrm{g} \mathrm{kg}^{-1}\right.$ corresponding to $30 \mu \mathrm{g} \mathrm{kg}^{-1}$ of Ang-(1-7)) by gavage. Blood samples were collected at 2, 4, 6, 8 and $24 \mathrm{~h}$ after treatment for measuring plasma Ang-(1-7) concentrations.

\subsection{Hemodynamic measurements}

Forty-eight hours after the last treatment day (10 weeks), rats from the chronic experiment were anesthetized with intramuscular injection of ketamine $(90 \mathrm{mg} / \mathrm{kg})$ and xylazine $(20 \mathrm{mg} / \mathrm{kg})$ for intra-arterial catheterization. Two polyethylene catheters (PE10, Biocorp Australia, Huntingdale, Victoria, Australia) filled with heparinized saline solution, connected to a strain gauge transducer (NarcoBiosystem Pulse Transducer RP-155, Houston, TX, USA) linked to a pressure amplifier (HP 8805C, Hewlett Packard, USA), were inserted into the femoral artery and vein for direct measurements of systolic, diastolic, mean arterial and pulse pressures (SAP, DAP, MAP and PP, respectively), heart rate (HR), and drug administration, respectively. The intra-arterial measurements were performed $48 \mathrm{~h}$ after the surgical procedure. After the conscious pressure recordings, rats were anesthetized through femoral vein catheter with sodium pentobarbital $(40 \mathrm{mg} / \mathrm{kg}$, i.v., Cristalia, SP, Brazil) and a PE-50 catheter was inserted through the right carotid artery and advanced into the LV for recording intraventricular systolic (LVSP) and end-diastolic (LVEDP) pressures, as well as the maximum rate of LV pressure rise and fall $(+\mathrm{d} P / \mathrm{d} T$ and $-\mathrm{d} P / \mathrm{d} T$ ). The obtained pressure recordings were taken using a microcomputer equipped with an analog-to-digital conversion board (Windaq, $2 \mathrm{kHz}$ sample rate, Dataq Instruments, Inc., Akron, $\mathrm{OH}, \mathrm{USA}$ ). Rats have their BP and HR constantly monitored during pentobarbital anesthesia to control the adequacy of the anesthesia. Intraventricular catheterization was only performed when BP and HR values were similar to those obtained with conscious intra-arterial recordings. An additional group of normotensive Wistar-Kyoto rats (25-week old) was added to the experiment to 
allow comparison of intraventricular pressures $(n=6)$. Immediately after LV recordings, rats were killed by decapitation still under anesthesia, to avoid neuro-hormonal changes in plasma and the heart. Signs of inflammation and pain were monitored after surgery and their absence justify avoiding the use of anti-inflammatory drugs in this experiment.

\subsection{Spectral analysis}

After detecting the pulse intervals, the heart period was automatically calculated on a beat-to-beat basis as the time interval between two consecutive systolic peaks or pulse interval (PI) in randomly chosen sequences of 200-300 beats [36]. Frequency domain analysis of HR variability and SAP variability were performed with an autoregressive algorithm on the PI interval sequences (tachogram) and on respective systolic sequences (systogram) [24]. The power spectral density was calculated for each time series. In this study, three spectral components were considered: very low frequency (VLF), from 0 to $0.25 \mathrm{~Hz}$; low frequency (LF) indicating sympathetic modulation, from 0.25 to $0.75 \mathrm{~Hz}$; and high frequency (HF) indicating parasympathetic modulation, from 0.75 to $3.00 \mathrm{~Hz}$. The spectral components were expressed in absolute units $\left(\mathrm{ms}^{2}\right.$ or $\mathrm{mm} \mathrm{Hg}^{2}$ ) [32].

\subsection{Plasma and cardiac Ang-(1-7) concentrations}

Blood samples were collected in tubes containing $1 \mathrm{mmol} / \mathrm{L}$ p-hydroxymercury benzoate, $30 \mathrm{mmol} / \mathrm{L}$ 1,10-phenanthroline, $1 \mathrm{mmol} / \mathrm{L}$ phenylmethylsulphonyl fluoride (PMSF), $1 \mathrm{mmol} / \mathrm{L}$ pepstatin A and 7.5\% EDTA. Samples were centrifuged at $250 \times \mathrm{g}$ for $10 \mathrm{~min}$, and plasma stored at $-80^{\circ} \mathrm{C}$. Approximately $200 \mathrm{mg}$ of LV were homogenized in $4 \mathrm{~mol} / \mathrm{L}$ of guanidine thiocyanate and $1 \%$ trifluoroacetic acid in water. Peptides were after extracted as previously described [29] using Bond-Elutphenylsilica cartridge (Varian, Inc., Palo Alto, CA, USA). After evaporation, plasma and cardiac Ang-(1-7) peptide immunoreactivity levels were measured by radioimmunoassay according to Botelho et al. [5]. Protein concentration in the heart was determined by the Bradford method [6].

\subsection{Histological analysis}

At the end of the treatment and exercise training protocol, the rats were killed by decapitation and the hearts were immediately removed, weighted and fixed in $10 \%$ formalin in $0.1 \mathrm{~mol} / \mathrm{L}$

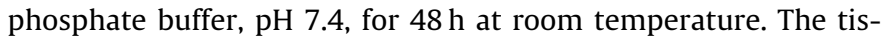
sue was embedded in paraffin, sectioned $(5 \mu \mathrm{m})$ and stained with hematoxylin and eosin. Images from 3 slides of each animal were captured and cardiac fibers from at least 80 cells of each group were measured using Leica microscopy using a $40 \times$ objective. Cardiomyocytes were analyzed only from longitudinal fibers with defined central nucleus.

\subsection{Statistical analysis}

Data are presented as mean \pm SEM. Comparisons between groups in the chronic experiment were performed using two-way ANOVA followed by Bonferroni test, considering Ang-(1-7) treatment and exercise training as two factors. Analysis of the cardiac Ang-(1-7) concentration used the non-parametric Kruskal-Wallis followed by Dunn's Multiple Comparison tests since the data did not presented normal distribution. The software GraphPad Prism version 5.00 for Windows (GraphPad Software, San Diego, CA, USA) was used for all tests performed. The significance level was established at $P<0.05$.

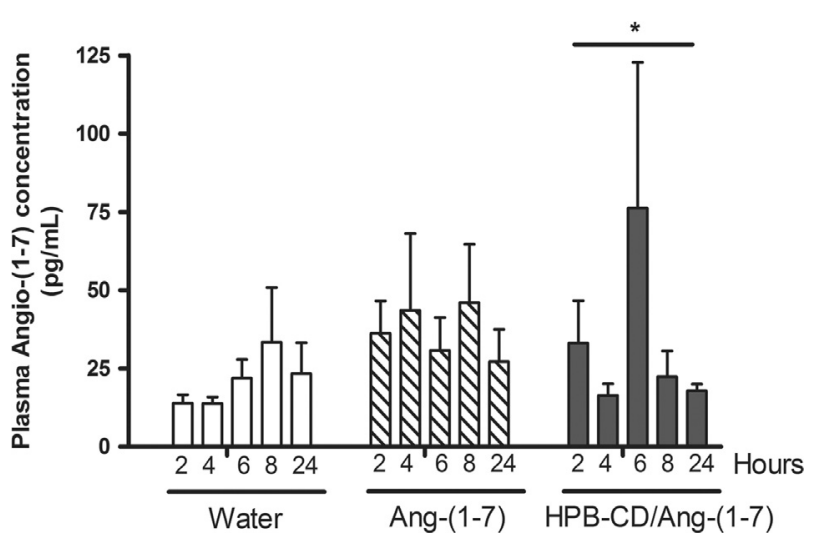

Fig. 1. Analysis of plasma Ang-(1-7) concentration after acute administrating of tap water, not included Ang-(1-7) and Ang-(1-7) included in hydroxy-prolyl-betacyclodextrin (HPB-CD) (HPB-CD/Ang-(1-7)). Data represent mean $\pm S E M .{ }^{*} P<0.05$ at $6 \mathrm{~h}$ in HPB-CD/Ang-(1-7) group compared to other times and treatments (repeatedmeasures ANOVA, $n=5$ /group).

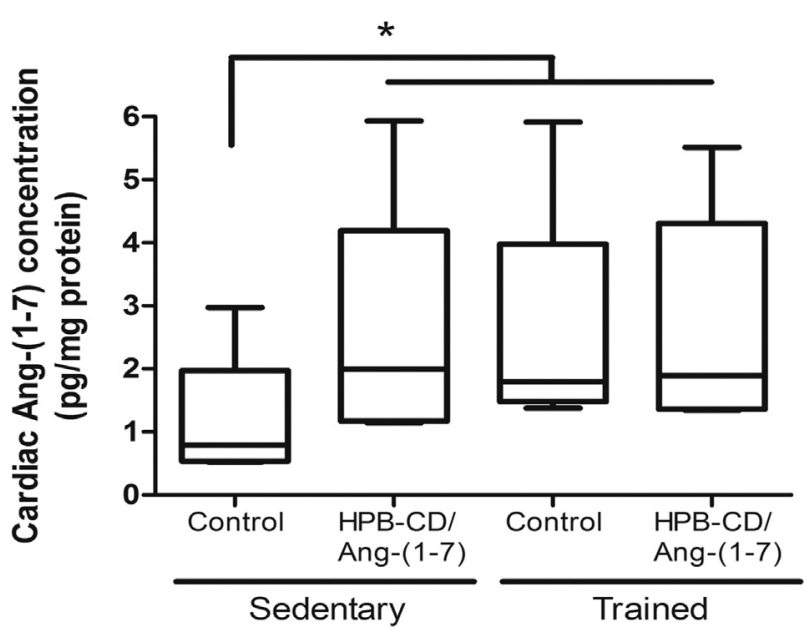

Fig. 2. Cardiac Ang-(1-7) concentration assessed by radioimmunoassay in sedentary and exercise-trained SHR, treated or not with orally active Ang-(1-7) included in hydroxy-prolyl-beta-cyclodextrin (HPB-CD) (HPB-CD/Ang-(1-7)). Data represent mean, minimum and maximum values analyzed by Kruskal-Wallis test ( $n=6 /$ group). ${ }^{*} P<0.05$ vs control sedentary group.

\section{Results}

\subsection{Acute Ang-(1-7) absorption}

Ang-(1-7) concentrations were assessed in plasma during $24 \mathrm{~h}$ after an acute administration of tap water (control), Ang-(1-7) not included and the HPB-CD/Ang-(1-7) inclusion compound by gavage. Fig. 1 shows that after $6 \mathrm{~h}$ of an acute administration, only the group treated with HPB-CD/Ang-(1-7) reached a peak of Ang-(1-7) concentration. The same profile was not observed in the group treated with Ang-(1-7) not included.

\subsection{Chronic $H P B-C D / A n g-(1-7)$ and exercise increased cardiac tissue Ang-(1-7) concentration}

Cardiac Ang-(1-7) concentration was assessed after chronic treatments by radioimmunoassay. Fig. 2 shows that all intervention groups have increased values of Ang-(1-7) concentration in the heart compared to the control group. 
Table 1

Blood pressure measurements in SHR pre and post-treatment with water (control) or HPB-CD/Ang-(1-7).

\begin{tabular}{|c|c|c|c|c|c|c|}
\hline & \multicolumn{2}{|l|}{ Sedentary } & \multicolumn{2}{|c|}{ Exercise-trained } & \multicolumn{2}{|c|}{ Two-way ANOVA } \\
\hline & Control & Ang-(1-7) & Control & Ang-(1-7) & Treatment & Training \\
\hline \multicolumn{7}{|c|}{ Pre-treatment tail cuff plethysmography } \\
\hline $\mathrm{SAP}(\mathrm{mm} \mathrm{Hg})$ & $208 \pm 3$ & $207 \pm 2$ & $200 \pm 2$ & $202 \pm 2$ & & \\
\hline $\mathrm{HR}(\mathrm{bpm})$ & $389 \pm 6$ & $390 \pm 4$ & $395 \pm 5$ & $394 \pm 6$ & & \\
\hline \multicolumn{7}{|c|}{ Post-treatment intraarterial BP } \\
\hline $\mathrm{SAP}(\mathrm{mm} \mathrm{Hg})$ & $223 \pm 3$ & $202 \pm 2^{\dagger}$ & $196 \pm 6^{\dagger}$ & $187 \pm 3^{\dagger}$ & *** & $* * *$ \\
\hline $\mathrm{DAP}(\mathrm{mm} \mathrm{Hg})$ & $162 \pm 3$ & $147 \pm 2$ & $138 \pm 5^{\dagger}$ & $128 \pm 6^{\dagger \ddagger}$ & $* *$ & $* * *$ \\
\hline MAP (mm Hg) & $192 \pm 2$ & $179 \pm 2^{\dagger}$ & $166 \pm 6^{\dagger}$ & $158 \pm 5^{\dagger \ddagger}$ & * & $* * *$ \\
\hline $\mathrm{PP}(\mathrm{mm} \mathrm{Hg})$ & $61 \pm 3$ & $55 \pm 1$ & $58 \pm 1$ & $59 \pm 3$ & & \\
\hline $\mathrm{HR}(\mathrm{bpm})$ & $339 \pm 12$ & $311 \pm 8^{\dagger}$ & $295 \pm 5^{\dagger}$ & $301 \pm 4^{\dagger}$ & & $* *$ \\
\hline
\end{tabular}

Values represent mean \pm SEM. Systolic arterial pressure, SAP; diastolic arterial pressure, DAP; mean arterial pressure, MAP; pulse pressure, PP; heart rate, HR.

${ }^{*} P<0.05$ on two-way ANOVA considering treatment (Ang-(1-7)) and training as factors.

** $P<0.01$ on two-way ANOVA considering treatment (Ang-(1-7)) and training as factors.

${ }^{* * *} P<0.001$ on two-way ANOVA considering treatment (Ang-(1-7)) and training as factors.

$\dagger P<0.05$ vs SHR sedentary control group.

$\ddagger P<0.05$ vs SHR sedentary treated (Ang-(1-7)) group.

\subsection{Chronic $H P B-C D / A n g-(1-7)$ and exercise-training reduced $B P$ and improved autonomic control}

Pre-treatment SAP and HR, measured by tail-cuff plethysmography, were not different between groups (Table 1). After treatment, reduced intra-arterial SAP, DAP, MAP and HR are observed in HPB-CD/Ang-(1-7) and in exercise-trained SHR (Table 1) and the association of both interventions potentiate their effects on BP.

Chronic treatment and exercise-training also improved cardiovascular autonomic control assessed by spectral analysis of intra-arterial BP recordings, indicating respectively the HR and SAP variabilities (Figs. 3 and 4). Significant improvement of HR variability was obtained with HPB-CD/Ang-(1-7) treatment, but not with exercise-training in SHR (Fig. 3A). However, both interventions ameliorated sympathetic (LF absolute, Fig. 3B) and parasympathetic (HF absolute, Fig. 3C) components of the HR variability. Both LF and HF data indicate an improvement of cardiac autonomic control in favor of the parasympathetic modulation after Ang-(1-7) treatment and exercise-training. Sympathovagal balance (LF/HF index, Fig. 3D) in the heart was also improved by both interventions.

Regarding SAP variability (Fig. 4A), similar beneficial effects were obtained after chronic HPB-CD/Ang-(1-7) treatment and exercise-training. Similar responses are also verified in both groups regarding sympathetic (Fig. 4B) and parasympathetic (Fig. 4C) components of the SAP variability, indicating the autonomic modulation on vessels. Absolute VLF (Fig. 4D) was also reduced by both interventions although its physiological meaning is still not well understood. Overall data show an improvement of autonomic control in the cardiovascular system obtained with HPB-CD/Ang-(1-7) treatment and exercise training, with both groups presenting very similar effects. No additive or synergistic effect was observed with the use of both interventions.

\subsection{Chronic $H P B-C D / A n g-(1-7)$ and exercise-training improved cardiac function}

Both interventions elicited similar changes in SHR LV function. Morphometric analysis of the heart is shown in Fig. 5. No difference in body weight was observed between control, HPB-CD/Ang-(1-7) treated and exercise-trained groups (Fig. 5A) although it is increased in the exercise-trained + treated group. Chronic HPB-CD/Ang-(1-7) reduced heart weight (Fig. 5B) and cardiac hypertrophy index, obtained from heart to body weight ratio (Fig. 5C), when compared to controls. Similar responses were observed with exercise training, whereas the association of Ang(1-7) treatment with exercise did not produced an additional effect.
Histological analysis shows that HPB-CD/Ang-(1-7) treatment has markedly decreased cardiomyocyte diameter compared to controls SHR (Fig. 5D and E). Exercise training, however, did not significantly decreased myocyte diameter compared to controls although its association with Angio-(1-7) was effective to reduce myocyte hypertrophy also compared to controls.

Chronic HPB-CD/Ang-(1-7) also improved LV function, here assessed by intraventricular catheterization to obtain LV enddiastolic and systolic pressures, as well as LV maximum and minimum derivatives $(\mathrm{d} P / \mathrm{d} T)$. Chronic treatment with the inclusion compound restored LV end-diastolic pressure in SHR to values similar to Wistar-Kyoto rats (normotensive strain as control for SHR) and exercise training had similar effect (Fig. 6A). A partial reduction in the LV systolic pressure was also observed with both interventions (Fig. 6B) although it remained higher compared to Wistar-Kyoto rats. Regarding the cardiac muscle mechanical properties, 25-week old SHR presented a compensatory cardiac hypertrophy with higher values of LV minimum and maximum $\mathrm{d} P / \mathrm{d} T$ in comparison to Wistar-Kyotos (Fig. 6C and D). However, chronic administration of the inclusion compound restores both derivatives to values similar to the Kyotos while exercise training only reduced the minimum $\mathrm{d} P / \mathrm{d} T$. Overall data indicate that chronic treatment with HPB-CD/Ang-(1-7) effectively improves LV function, mainly by restoring LV diastolic function and mechanical properties of contractility and relaxation. HPB-CD/Ang-(1-7) and exercise training had similar effects.

\section{Discussion}

In the present study, we report that chronic treatment with the inclusion compound HPB-CD/Ang-(1-7) is effective in reducing BP and improving overall cardiovascular autonomic control in SHR. The inclusion compound also attenuated cardiac hypertrophy and restored LV function, presenting similar effects to those produced by exercise training.

We observed that both exercise training and chronic oral administration of the Ang-(1-7) inclusion compound elicited a significant reduction of BP values (around $15-20 \mathrm{~mm} \mathrm{Hg}$ in SHR). Enhanced autonomic modulation of the cardiovascular system was also evident in trained or treated SHR. This was achieved mainly by decreased sympathetic modulation on heart and vessels and increased parasympathetic modulations on heart after both interventions, which may directly contribute to reduce BP in both groups. Overall data indicate beneficial effects of both HPB-CD/Ang-(1-7) treatment and exercise training on cardiovascular system although the combination of both interventions did 
A

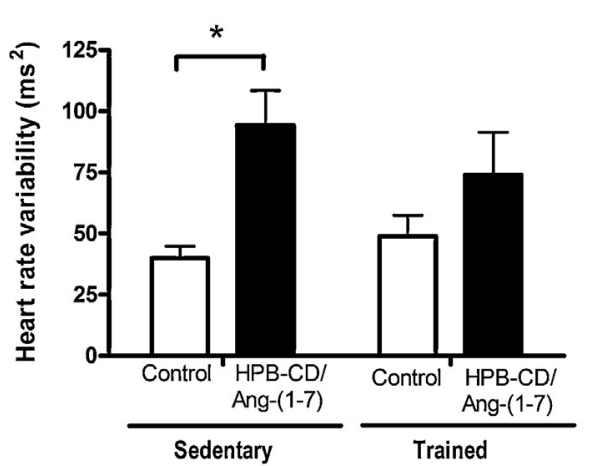

C

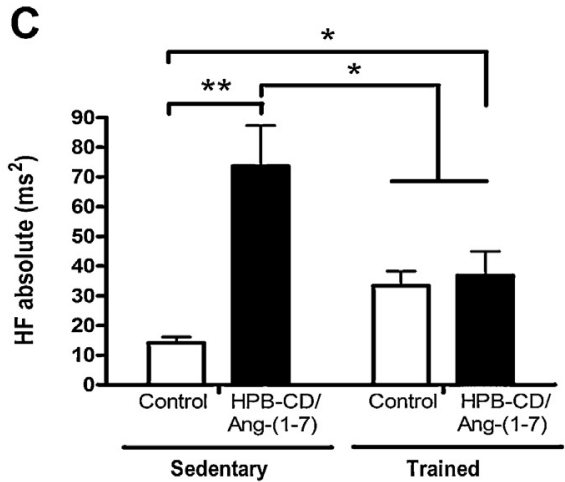

B

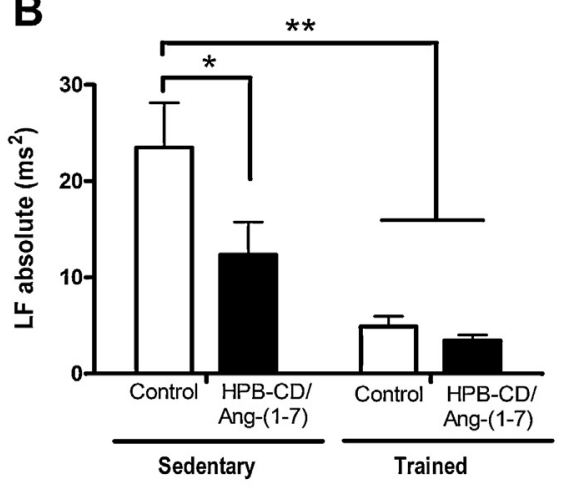

D

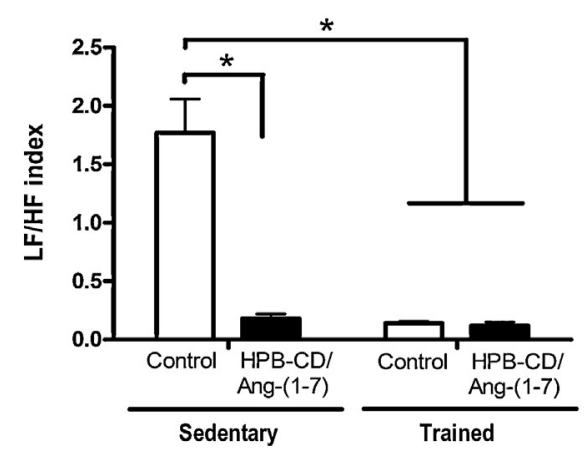

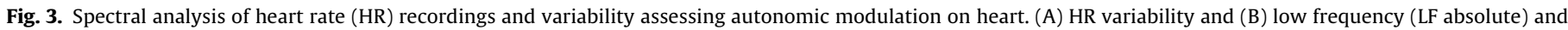

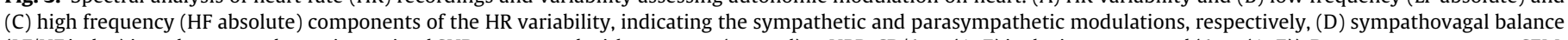

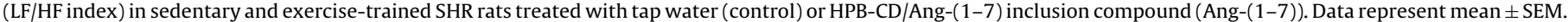
${ }^{*} P<0.05$ and ${ }^{* *} P<0.01$ vs group indicated, two-way ANOVA ( $n=8$ /group).

\section{A}

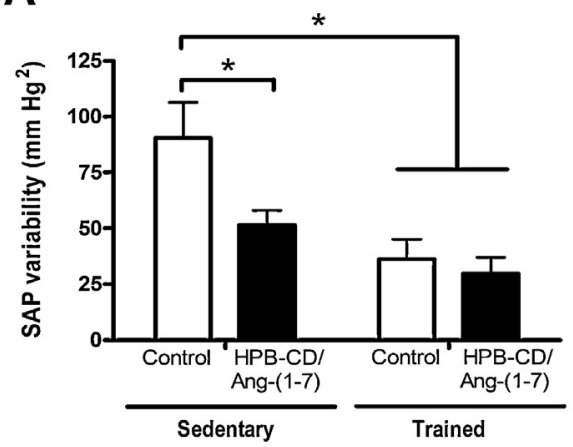

C

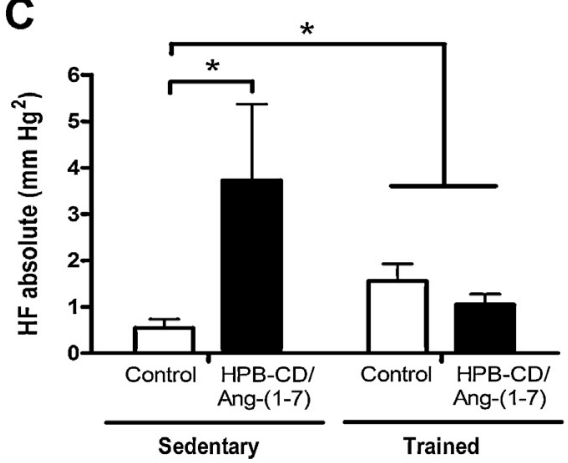

B

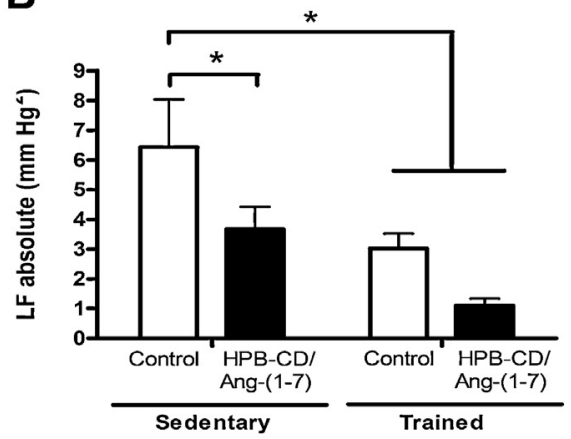

D

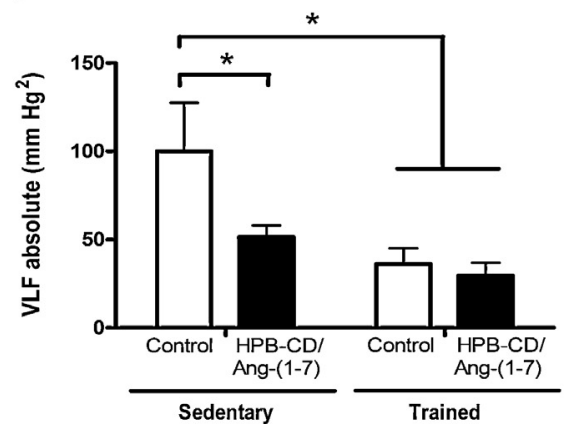

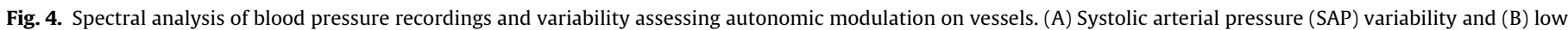

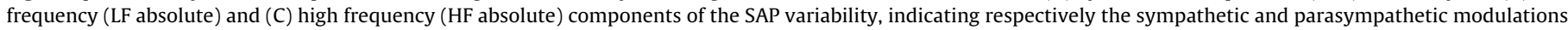

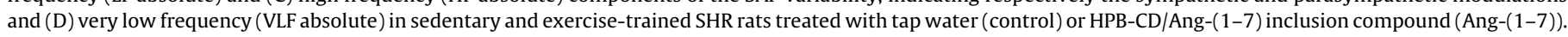
Data represent mean \pm SEM. ${ }^{*} P<0.05$ vs group indicated, two-way ANOVA ( $n=8 /$ group). 
A

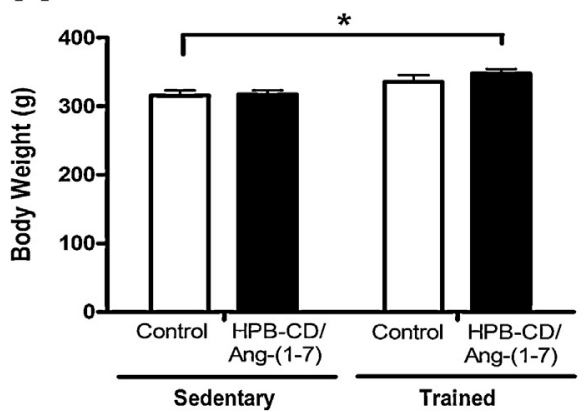

B

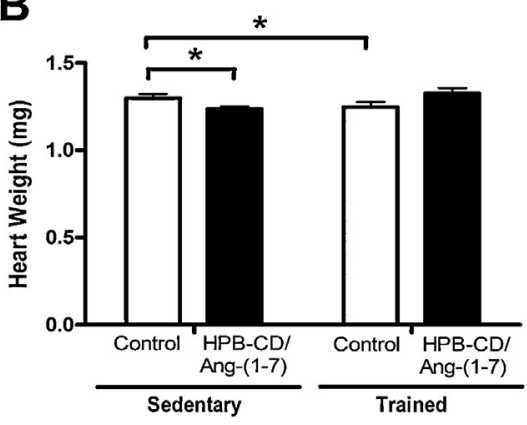

C

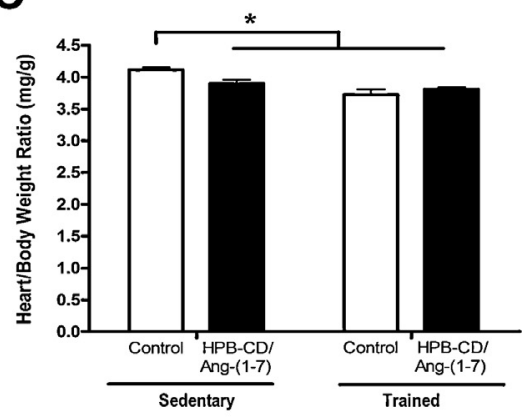

D

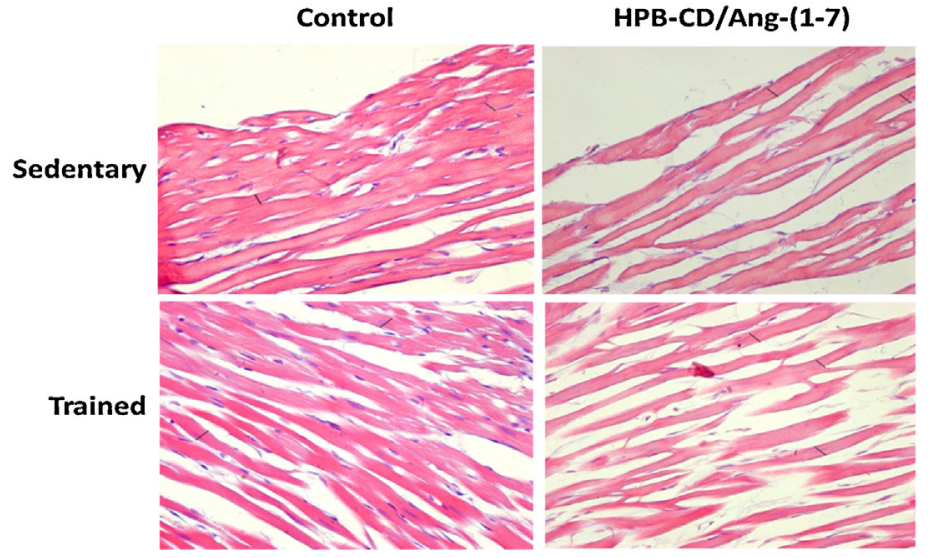

E

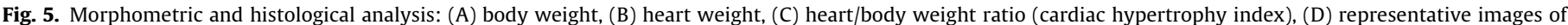

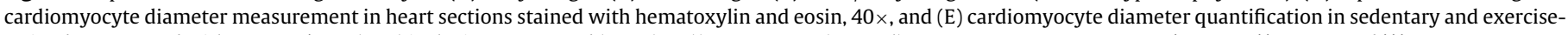

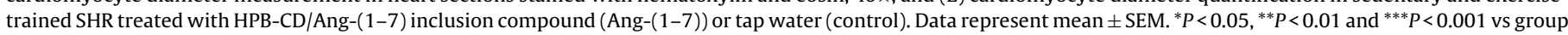
indicated, two-way ANOVA ( $n=8 /$ group).

not potentiate their isolated responses in SHR. This later observation might suggest that changes in Ang-(1-7) concentration play a role in the beneficial effects of exercise. Indeed, our study shows that both inclusion compound-treated and exercise-trained groups have higher cardiac concentrations of Ang-(1-7). Increased LV Ang-(1-7) concentration induced by exercise was also previously reported in SHR after swimming training [15] and it might exerts a key role in preventing cardiac remodeling and dysfunction in this model.

Different experimental strategies are being currently tested for stimulation of the ACE2/Ang-(1-7)/Mas axis in animal models of cardiovascular diseases [14]. In isoproterenol-induced cardiac dysfunction, treatment with AVE 0991, a nonpeptide Mas agonist, attenuated cardiac dysfunction and fibrosis [13]. Treatment with AVE 0991 improved cardiac function, assessed in vitro, by restoring overall cardiac muscle mechanical properties of contractility and relaxation. AVE 0991 also ameliorated cardiac function in rats submitted to myocardial infarction [12]. Genetic approaches targeting ACE2/Ang-(1-7)/Mas axis also provided evidence for a cardioprotective role of Ang-(1-7) [30,41]. More recently it has been shown that transgenic overexpression of Ang-(1-7) prevented the development of LV diastolic dysfunction after induction of DOCA-salt hypertension [38]. A cardioprotective effect of ACE2/Ang-(1-7)/Mas axis has been also suggested by the use of putative ACE2 activators, such as xanthenone and resorcinolnaphthalein [18].

In our study, we tested a novel orally active formulation of Ang-(1-7), the inclusion compound of HPB-CD/Ang-(1-7) [23], in a low dosage (equivalent to $30 \mu \mathrm{g} \mathrm{kg}^{-1} \mathrm{day}^{-1}$ of the peptide), as another therapeutic approach for hypertension-induced cardiovascular changes. For this purpose, we first evaluated the pharmacokinetic of the inclusion compound in SHR. In SHR, the peptide reached a peak of plasma Ang-(1-7) concentrations at $6 \mathrm{~h}$ after oral administration. The curve here obtained by treating SHR with the inclusion compound was similar to that previously observed in Wistar rats [26]. The dose used was effective in increasing cardiac Ang-(1-7) concentrations to values similar to those obtained with exercise training. The effectiveness of the Ang-(1-7) oral formulation is also evident by the marked changes in HR and SAP variability and autonomic modulation. Our data are in keeping with recent data from our group showing the effectiveness of this formulation in myocardial infarction [26] and as anti-trombogenic [16]. Importantly, the inclusion compound did not present any adverse effect in SHR as well as in other animal models previously studied. This is in agreement with the fact that other cyclodextrin inclusion compounds have been used in human beings without adverse effects [9].

In SHR and in human hypertension, an imbalance between sympathetic and parasympathetic modulations on cardiovascular system impairs the regulation of $\mathrm{BP}$, and in the heart, it impacts in cardiac muscle inotropy and chronotropy. Our group has previously shown that exercise training in SHR decreases sympathetic modulation and improves SAP variability, which was associated with decreased cardiac norepinephrine concentrations [4]. Additionally, it is also well described that exercise training improves baroreflex sensitivity and cardiovascular autonomic control in SHR which was also correlated with the improvement of the peripheral and vascular oxidant stress profile [3]. We here observed a significant improvement of SAP and HR variability in trained SHR. Chronic treatment with Ang-(1-7) decreased the sympathetic modulation on heart and vessels ( LF components obtained from $\mathrm{HR}$ and SAP). Ang-(1-7) treatment has, in addition, increased the HF 

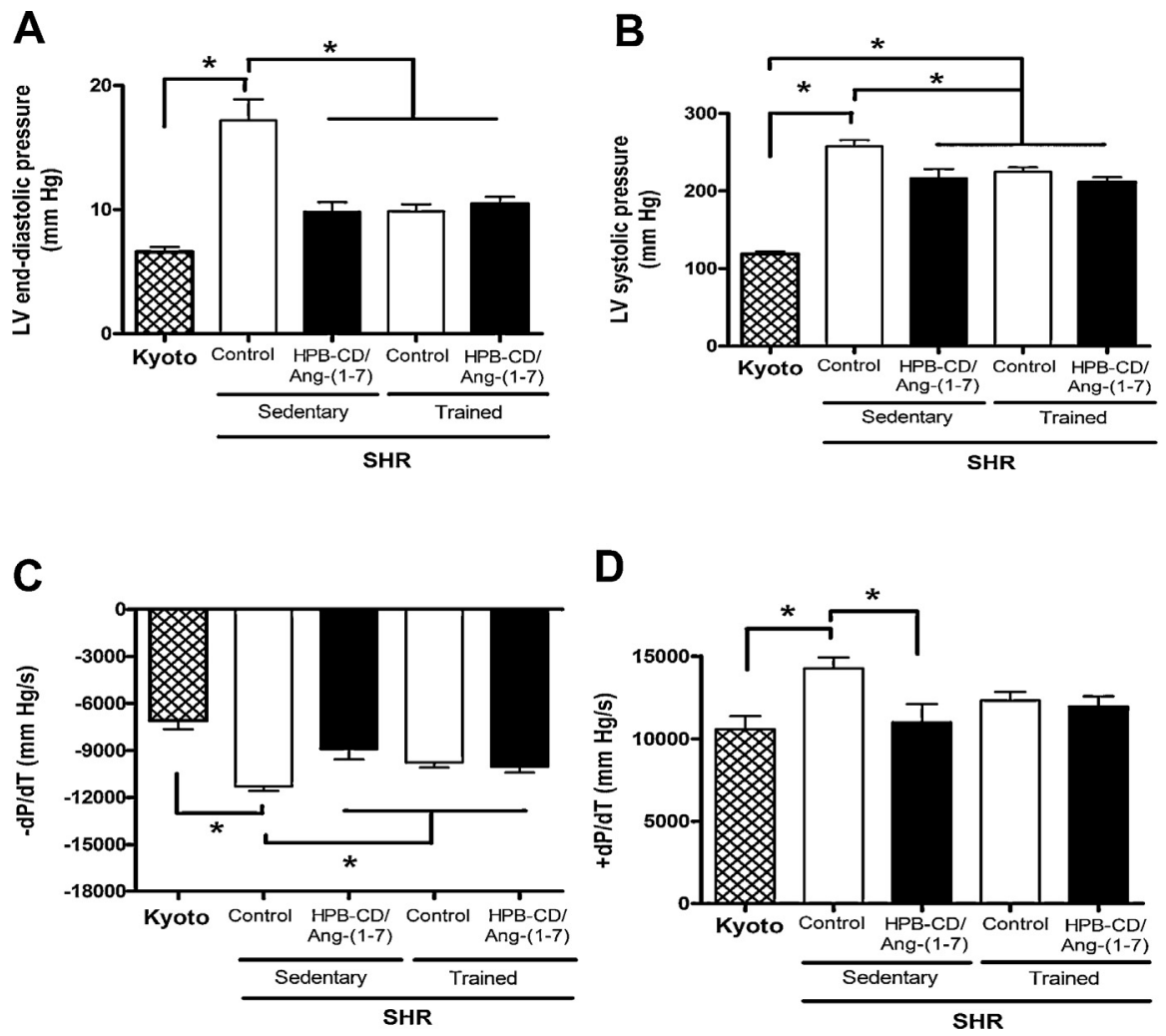

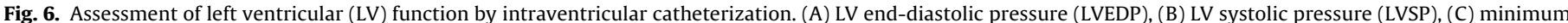

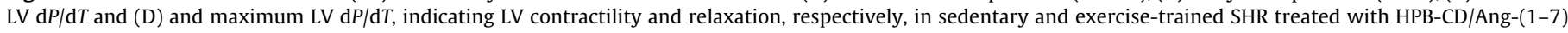

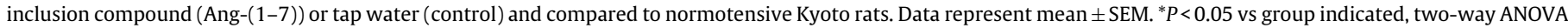
( $n=6 /$ group).

component, indicating an increment of the parasympathetic activity in cardiovascular system, being more significant in the heart. By comparing both interventions used in our study, we therefore show that while Ang-(1-7) has a significant impact in ameliorating parasympathetic modulation, exercise is more effective in reducing sympathetic modulation in SHR hearts. Whether autonomic responses elicited by orally active Ang-(1-7) are caused systemically or locally in heart and vessels was not addressed in our study, and indeed, both possibilities are corroborated by others [18,19]. Previously, Xiao et al. [42] observed that central overexpression of ACE2 decreased norepinephrine release and the renal sympathetic nerve activity in mice with heart failure. Byku et al. [7] have also shown that peripherally, Ang-(1-7) decreased nerve-stimulated norepinephrine overflow and perfusion pressure in the mesenteric arterial bed of SHR. On the other hand, data showing Ang-(1-7)-induced modulation of cardiovascular sympathetic and parasympathetic activities, as well as sympathovagal balance (LF/HF index), suggest a central effect of this peptide on $\mathrm{BP}$ regulation. In keeping with our results, a previous study from Heringer-Walther et al. [17], also performed in SHR, has shown that Ang-(1-7) is involved in the central autonomic regulation of BP by modulating sympathetic neurons. Further, in rabbits with pacing-induced cardiac heart failure, central infusion of Ang-(1-7) blunted sympathetic outflow and stimulated vagal outflow [19]. These observations favor the hypothesis that our results could be at least partially dependent on a central action of Ang-(1-7), conceivably acting primarily in brain-barrier deficient regions. Further studies are obviously needed to confirm this possibility.

Beneficial effects of Ang-(1-7) on autonomic modulation as shown here forHR and SAP variabilities, probably contribute for restoring cardiac function and lowering $\mathrm{BP}$, here observed in rats treated with the inclusion compound. In this regard, chronic treatment with Ang-(1-7) was very similar to exercise training effects in restoring cardiovascular autonomic control. The results obtained with HPB-CD/Ang-(1-7) and exercise training may also have future implications on clinical outcomes in hypertensive population since HR and SAP variability are considered predictors of cardiac events in healthy people and in patients with cardiovascular diseases $[21,25,35]$.

Regarding cardiac changes induced by both treatments, beneficial effects obtained after chronic treatment with HPBCD/Ang-(1-7) in SHR comprised a diminished cardiac hypertrophy and improved function. Similar results were also verified using the same compound in models of myocardial infarction and isoproterenol-induced heart failure [26]. Amelioration of cardiac remodeling caused by HPB-CD/Ang-(1-7) treatment and exercise training is accompanied by the improvement of LV function in SHR. We showed that chronic treatment with the inclusion compound restores LV end-diastolic pressure to values similar to normotensive Wistar-Kyoto rats. Exercise training caused similar responses in both LV pressures. Mechanical improvement of the cardiac muscle is also shown by LV maximum and minimum derivatives. We demonstrated that only Ang-(1-7) treatment restores both derivatives of contractility and relaxation, whereas exercisetraining ameliorated only the relaxation component. Alterations in the autonomic control on heart caused by both treatments may contribute to improve cardiac muscle mechanical properties.

\subsection{Conclusion}

In conclusion, chronic administration of HPB-CD/Ang-(1-7) inclusion compound in SHR decreases BP and greatly improves the autonomic modulation of the cardiovascular system. Beneficial cardiovascular responses obtained with the orally active Ang-(1-7) 
compound also include the improvement of LV function and cardiac muscle mechanical properties. Treatment with the Ang(1-7) inclusion compound produced responses similar to those of exercise training mainly on attenuating cardiac hypertrophy and enhancing cardiovascular autonomic control. Our data reinforces the feasibility of the use of an orally active HPB-CD/Ang-(1-7) inclusion compound for managing BP and target-organ damage induced by hypertension.

\subsection{Perspectives}

The results obtained with HPB-CD/Ang-(1-7) treatment highlight the potential therapeutic effect of an orally active Ang-(1-7) formulation as a putative mimetic of exercise training on handling the deleterious cardiac dysfunction induced by hypertension. We used a rather low effective once-a-day dose of Ang-(1-7), which has been used in a previous study examining the antithrombotic effect of Ang-(1-7) in SHR [16] and its cardioprotective effect in myocardial infarction [27] and isoproterenol-induced cardiac dysfunction and remodeling [26].

However, despite of the dramatic effects observed in the current study in the diastolic dysfunction and HR variability, in future studies would be important to evaluate the effect of different doses of the inclusion compound, especially on BP since Ang-(1-7) is known as a vasodilator, as well as to compare the hemodynamic effects of this compound with other anti-hypertensive drugs. A more detailed assessment of the mechanism by which the Ang(1-7) is absorbed in the digestive tract, as well as whether this peptide crosses the encephalic barrier and/or penetrate cardiac tissue, will be also important in order to determine the mechanism of the exercise-mimetic activity of Ang-(1-7) in the cardiovascular system.

\section{Acknowledgements}

The authors acknowledge the expert technical assistance of Marilene Luiza de Oliveira and José Roberto da Silva.

This work was supported by Institutos Nacionais de Ciência e Tecnologia (INCT) project Instituto Nacional de C\&T en Nanobiofarmacêutica (N-BIOFAR) from Minas Gerais, Conselho Nacional de Desenvolvimento Científico e Tecnológico (CNPq), Fundação de Amparo a Pesquisa do Estado de Minas Gerais (FAPEMIG) and Fundação de Amparo a Pesquisa do Estado do Rio Grande do Sul (FAPERGS). M.B. was recipient of a postdoctoral fellowship from Programa Nacional de Cooperação Acadêmica (PROCAD) and Programa Nacional de Pos-Doutorado (PNPD) of Conselho de Aperfeiçoamento de Pessoal de Nivel Superior (CAPES).

\section{References}

[1] Anavekar NS, Solomon SD. Angiotensin II receptor blockade and ventricular remodelling. J Renin Angiotensin Aldosterone Syst 2005;6:43-8.

[2] Barauna VG, Magalhaes FC, Krieger JE, Oliveira EM. AT1 receptor participates in the cardiac hypertrophy induced by resistance training in rats. Am J Physiol Regul Integr Comp Physiol 2008;295:R381-7.

[3] Bertagnolli M, Campos C, Schenkel P, de Oliveira V, De Angelis K, BellóKlein A, et al. Baroreflex sensitivity improvement is associated with decreased oxidative stress in trained spontaneously hypertensive rat. J Hypertens 2006;24:2437-43.

[4] Bertagnolli M, Schenkel P, Campos C, Mostarda C, Casarini D, Belló-Klein A, et al. Exercise training reduces sympathetic modulation on cardiovascular system and cardiac oxidative stress in spontaneously hypertensive rats. Am J Hypertens 2008:21:1188-93.

[5] Botelho L, Block C, Khosla M, Santos R. Plasma angiotensin(1-7) immunoreactivity is increased by salt load, water deprivation, and hemorrhage. Peptides 1994;15:723-9.

[6] Bradford M. A rapid and sensitive method for the quantitation of microgram quantities of protein utilizing the principle of protein-dye binding. Anal Biochem 1976;72:248-54.
[7] Byku M, Macarthur H, Westfall TC. Inhibitory effects of angiotensin-(1-7) on the nerve stimulation-induced release of norepinephrine and neuropeptide Y from the mesenteric arterial bed. Am J Physiol Heart Circ Physiol 2010;298:H457-65.

[8] Chicco AJ, McCune SA, Emter CA, Sparagna GC, Rees ML, Bolden DA, et al. Lowintensity exercise training delays heart failure and improves survival in female hypertensive heart failure rats. Hypertension 2008;51:1096-102.

[9] Davis ME, Brewster ME. Cyclodextrin-based pharmaceutics: past, present and future. Nat Rev Drug Discov 2004;3:1023-35.

[10] Ferrario C. Angiotensin-converting enzyme 2 and angiotensin-(1-7): an evolving story in cardiovascular regulation. Hypertension 2006;47:515-21.

[11] Ferrario C, Trask A, Jessup J. Advances in biochemical and functional roles of angiotensin-converting enzyme 2 and angiotensin-(1-7) in regulation of cardiovascular function. Am J Physiol Heart Circ Physiol 2005;289:H2281-90.

[12] Ferreira A, Jacoby B, Araújo C, Macedo F, Silva G, Almeida A, et al. The nonpeptide angiotensin-(1-7) receptor Mas agonist AVE-0991 attenuates heart failure induced by myocardial infarction. Am J Physiol Heart Circ Physiol 2007;292:H1113-9.

[13] Ferreira A, Oliveira T, Castro M, Almeida A, Castro C, Caliari M, et al. Isoproterenol-induced impairment of heart function and remodeling are attenuated by the nonpeptide angiotensin-(1-7) analogue AVE 0991. Life Sci 2007;81:916-23.

[14] Ferreira AJ, Bader M, Santos RA. Therapeutic targeting of the angiotensinconverting enzyme 2/Angiotensin-(1-7)/Mas cascade in the renin-angiotensin system: a patent review. Expert Opin Ther Pat 2012;22:567-74.

[15] Filho A, Ferreira A, Santos S, Neves S, Silva Camargos E, Becker L, et al. Selective increase of angiotensin(1-7) and its receptor in hearts of spontaneously hypertensive rats subjected to physical training. Exp Physiol 2008;93:589-98

[16] Fraga-Silva RA, Costa-Fraga FP, De Sousa FB, Alenina N, Bader M, Sinisterra RD, et al. An orally active formulation of angiotensin-(1-7) produces an antithrombotic effect. Clinics (Sao Paulo) 2011;66:837-41.

[17] Heringer-Walther S, Batista EN, Walther T, Khosla M, Santos R, CampagnoleSantos M. Baroreflex improvement in shr after ace inhibition involves angiotensin-(1-7). Hypertension 2001;37:1309-14

[18] Hernández Prada JA, Ferreira AJ, Katovich MJ, Shenoy V, Qi Y, Santos RA, et al. Structure-based identification of small-molecule angiotensinconverting enzyme 2 activators as novel antihypertensive agents. Hypertension 2008; $51: 1312-7$

[19] Kar S, Gao L, Belatti DA, Curry PL, Zucker IH. Central angiotensin (1-7) enhances baroreflex gain in conscious rabbits with heart failure. Hypertension 2011;58:627-34.

[20] Krieger E, Brum P, Negrão C. State-of-the-Art lecture: influence of exercise training on neurogenic control of blood pressure in spontaneously hypertensive rats. Hypertension 1999;34:720-3

[21] La Rovere M, Bigger JJ, Marcus F, Mortara A, Schwartz P. Baroreflex sensitivity and heart-rate variability in prediction of total cardiac mortality after myocardial infarction. ATRAMI (Autonomic Tone and Reflexes After Myocardial Infarction) Investigators. Lancet 1998;351:478-84.

[22] Loot A, Roks A, Henning R, Tio R, Suurmeijer A, Boomsma F, et al. Angiotensin(1-7) attenuates the development of heart failure after myocardial infarction in rats. Circulation 2002;105:1548-50.

[23] Lula I, Denadai A, Resende J, de Sousa F, de Lima G, Pilo-Veloso D, et al. Study of angiotensin-(1-7) vasoactive peptide and its beta-cyclodextrin inclusion complexes: complete sequence-specific NMR assignments and structural studies. Peptides 2007;28:2199-210.

[24] Malliani A, Pagani M, Lombardi F, Cerutti S. Cardiovascular neural regulation explored in the frequency domain. Circulation 1991;84:482-92.

[25] Mancia G, Omboni S, Parati G. The importance of blood pressure variability in hypertension. Blood Press Monit 2000;5(Suppl. 1):S9-15.

[26] Marques FD, Ferreira AJ, Sinisterra RD, Jacoby BA, Sousa FB, Caliari MV, et al. An oral formulation of angiotensin-(1-7) produces cardioprotective effects in infarcted and isoproterenol-treated rats. Hypertension 2011;57: 477-83.

[27] Marques FD, Melo MB, Souza LE, Irigoyen MC, Sinisterra RD, de Sousa FB, et al. Beneficial effects of long-term administration of an oral formulation of Angiotensin-(1-7) in infarcted rats. Int J Hypertens 2012;2012:795452.

[28] Martin JE, Dubbert PM, Cushman WC. Controlled trial of aerobic exercise in hypertension. Circulation 1990;81:1560-7.

[29] Mendes A, Ferreira A, Pinheiro S, Santos R. Chronic infusion of angiotensin-(1-7) reduces heart angiotensin II levels in rats. Regul Pept 2005;125:29-34.

[30] Mercure C, Yogi A, Callera GE, Aranha AB, Bader M, Ferreira AJ, et al Angiotensin(1-7) blunts hypertensive cardiac remodeling by a direct effect on the heart. Circ Res 2008;103:1319-26.

[31] Molmen-Hansen HE, Stolen T, Tjonna AE, Aamot IL, Ekeberg IS, Tyldum GA, et al. Aerobic interval training reduces blood pressure and improves myocardial function in hypertensive patients. Eur J Prev Cardiol 2012;19: 151-60.

[32] Montano N, Ruscone T, Porta A, Lombardi F, Pagani M, Malliani A. Power spectrum analysis of heart rate variability to assess the changes in sympathovagal balance during graded orthostatic tilt. Circulation 1994;90:1826-31.

[33] Moraes-Teixeira Jde A, Felix A, Fernandes-Santos C, Moura AS, Mandarim-deLacerda CA, de Carvalho JJ. Exercise training enhances elastin, fibrillin and nitric oxide in the aorta wall of spontaneously hypertensive rats. Exp Mol Pathol 2010;89:351-7.

[34] Nunez E, Hosoya K, Susic D, Frohlich ED. Enalapril and losartan reduced cardiac mass and improved coronary hemodynamics in SHR. Hypertension 1997;29:519-24. 
[35] Parati G, Ulian L, Santucciu C, Omboni S, Mancia G. Blood pressure variability, cardiovascular risk and antihypertensive treatment. J Hypertens Suppl 1995; 13:S27-34.

[36] Porta C, Barone M, Bressan M. Rhe irreplaceable image: superficial thrombosis of a varicose vein of the abdominal wall as the first sign of an otherwise occult locally-advanced ovarian cancer. Haematologica 2001;86:1120.

[37] Sano H, Okamoto H, Kitabatake A, Iizuka K, Murakami T, Kawaguchi H. Increased mRNA expression of cardiac renin-angiotensin system and collagen synthesis in spontaneously hypertensive rats. Mol Cell Biochem 1998;178:51-8.

[38] Santiago N, Guimarães P, Sirvente R, Oliveira L, Irigoyen M, Santos R, et al. Lifetime overproduction of circulating Angiotensin-(1-7) attenuates deoxycorticosterone acetate-salt hypertension-induced cardiac dysfunction and remodeling. Hypertension 2010;55:889-96.

[39] Santos R, Ferreira A, Simões E, Silva A. Recent advances in the angiotensinconverting enzyme 2-angiotensin(1-7)-Mas axis. Exp Physiol 2008;93:519-27.
[40] Santos R, Simoes e Silva A, Maric C, Silva D, Machado R, de Buhr I, et al. Angiotensin-(1-7) is an endogenous ligand for the $G$ protein-coupled receptor Mas. Proc Natl Acad Sci USA 2003;100:8258-63.

[41] Santos RA, Ferreira AJ, Nadu AP, Braga AN, de Almeida AP, Campagnole-Santos $\mathrm{MJ}$, et al. Expression of an angiotensin-(1-7)-producing fusion protein produces cardioprotective effects in rats. Physiol Genomics 2004;17:292-9.

[42] Xiao Y, Zhou S, Liu Q. Ablation points of renal sympathetic denervation: the more, the better? Hypertension 2012;60:e47.

[43] Xie HH, Shen FM, Zhang XF, Jiang YY, Su DF. Blood pressure variability, baroreflex sensitivity and organ damage in spontaneously hypertensive rats treated with various antihypertensive drugs. Eur J Pharmacol 2006;543: 77-82.

[44] Yamamoto K, Masuyama T, Sakata Y, Mano T, Nishikawa N, Kondo H, et al. Roles of renin-angiotensin and endothelin systems in development of diastolic heart failure in hypertensive hearts. Cardiovasc Res 2000;47:274-83. 